Running head: Approach motivation in cerebral cortex

IN PRESS - PLEASE CITE AS:

Brookshire, G. \& Casasanto, D. (2018). Approach motivation in human cerebral cortex. Philosophical Transactions of the Royal Society B. DOI: 10.1098/rstb.2017-0141

\title{
Approach motivation in human cerebral cortex
}

Geoffrey Brookshire and Daniel Casasanto

Cornell University

University of Chicago

Word count: 4817 words (main text), 189 words (abstract)

Author Note

Geoffrey Brookshire, Department of Human Development, Cornell University; Department of Psychology, University of Chicago; Daniel Casasanto, Departments of Human Development and Psychology, Cornell University; Department of Psychology, Grossman Institute for Neuroscience, University of Chicago.

We thank Cleve Graver (C.G.) for his assistance in data collection. This document has benefitted from helpful comments from Sian Beilock, Marc Berman, Susan Goldin-Meadow, Richard B. Ivry, Marcel Kinsbourne, and members of the Experience and Cognition Laboratory. G.B. and D.C. designed the study and wrote the paper. G.B. and C.G. collected the data, and G.B. analyzed the data. This research was supported by a fellowship from the William Orr Dingwall Foundation to G.B. and by an NSF grant (BCS \#125710) and a James S. McDonnell Foundation Scholar Award (\#220020236) to D.C. A preliminary version of this study was presented at the 35th Annual Conference of the Cognitive Science Society, Berlin, DE.

Correspondence concerning this article should be addressed to Daniel Casasanto (casasanto@alum.mit.edu). 


\begin{abstract}
Different regions of the human cerebral cortex are specialized for different emotions, but the principles underlying this specialization have remained unknown. According to the sword and shield hypothesis, hemispheric specialization for affective motivation, a basic dimension of human emotion, varies across individuals according to the way they use their hands to perform approach- and avoidance-related actions. In a test of this hypothesis, here we measured approach motivation before and after 5 sessions of transcranial direct current stimulation (tDCS) to increase excitation in the left or right dorsolateral prefrontal cortex (DLPFC), in healthy adults whose handedness ranged from strongly left-handed to strongly right-handed. The strength and direction of participants' handedness predicted whether electrical stimulation to frontal cortex caused an increase or decrease in their experience of approach-related emotions. The organization of approach motivation in the human cerebral cortex varies across individuals as predicted by the organization of the individuals' motor systems. These results show that the large-scale cortical organization of abstract concepts corresponds with the way people use their hands to interact with the world. Affective motivation may reuse neural circuits that evolved for performing approach- and avoidance-related motor actions.
\end{abstract}

Keywords: approach motivation; emotion; hemispheric specialization; sword and shield hypothesis; tDCS. 
Approach motivation in human cerebral cortex

Emotions are a paradigm case of abstract concepts. You can never see happiness, even if you can see a smile, or touch anger, even if you can feel your face get flushed. Yet, people's abstract emotions appear to be grounded in their concrete interactions with the physical world in multiple ways [1]. Here we show that the cerebral organization of affective motivation, a basic dimension of human emotions, is predicted by the way people use their hands to perform approach and avoidance actions.

Affective motivation is the predisposition to approach or avoid physical or social stimuli. According to more than a hundred studies, left fronto-temporal cortex subserves approachrelated emotions like happiness and anger, whereas right fronto-temporal cortex subserves avoidance-related emotions like disgust and fear [2-7]. Although this pattern is well supported by data, the functional principles that give rise to the observed cortical specialization for emotions have remained unclear. Links between action and emotion suggest a possible organizing principle.

People often use their dominant hand for approach actions, and their non-dominant hand for avoidance actions [8-10]. For instance, people tend to use the dominant hand to grab a small ball (an approach action), but raise the non-dominant hands reflexively to protect themselves if a ball is thrown at them unexpectedly (an avoidance action [10]). In an iconic illustration of these action tendencies, swordsmen in centuries past wielded the sword in their dominant hand to attack the enemy (an approach action), and raised the shield with their non-dominant hand to fend off attack (an avoidance action).

Putting together this "sword-and-shield" pattern of hand actions with the results of numerous studies of motivation in the brain, we observed that, for right-handers, the hemisphere 
that controls the hand preferred for approach actions is also specialized for approach motivation, and the hemisphere that controls the hand preferred for avoidance actions is specialized for avoidance motivation. This may be no mere coincidence.

According to the sword and shield hypothesis [8], affective motivation in the cerebral cortex depends on neural systems for planning and performing motivated actions with the dominant and non-dominant hands. Specifically, we hypothesized that the cortical substrates of approach motivation overlap functionally and anatomically with cortical circuits for performing approach actions, and the cortical substrates of avoidance motivation with circuits for performing avoidance actions. In previous studies (see ref. 6 for a review), approach-related cortical activity may have been found in the left hemisphere not because the left hemisphere is inherently specialized for approach motivation, but rather because almost every study has tested exclusively right-handed subjects, who tend to perform approach actions with their right hand (i.e., their "sword hand") and avoidance actions with their left (i.e., their "shield hand"). This proposal predicts that the well-established pattern of hemispheric specialization for approach emotions may only obtain for strong right-handers, and that individual differences in manual motor control should correspond to differences in cortical specialization for motivation.

In a previous study [8], we tested the sword and shield hypothesis using electroencephalography (EEG). Experiments in right-handers have shown that people with stronger trait approach motivation have relatively higher left-hemisphere activation at rest [1113]. We predicted that this relationship should reverse in left-handers. Consistent with this prediction, we found that stronger approach motivation corresponded to greater left-hemisphere activity in right-handers, but corresponded to greater right-hemisphere activity in left-handers. A significant difference in the laterality of approach motivation in left- vs. right-handers was 
observed robustly, at 10 different pairs of homologous right- and left-hemisphere electrodes, including a pair of superior frontal electrodes (near F3-F4) where approach-related EEG asymmetries have been observed most frequently in right-handers [8].

The current study tested whether the lateralization of approach motivation varies continuously with the strength and direction of individuals' handedness. If there is a functional relationship between the cortical substrates of approach motivation and of motor control for approach-related actions, which are performed preferentially with the dominant hand, then: (a) the hemispheric laterality of approach motivation should reverse between strong left-handers and strong right-handers, as in our earlier EEG study, and (b) graded differences in handedness should correspond to graded differences in the lateralization of approach motivation.

To test these predictions, we measured approach motivation before and after five daily 20-minute sessions of transcranial direct current stimulation (tDCS). In tDCS, a weak electrical current is passed through the cortex by electrodes on the scalp, leading to modulations in excitability of neurons beneath the electrodes [14]. In a double-blind procedure, we used tDCS to increase neural excitability in either the left or right frontal cortex for each participant. We analyzed changes in self-reported approach motivation as a function of participants' handedness (measured continuously), and of the polarity of tDCS stimulation they received (leftanodal/right-cathodal or right-anodal/left-cathodal).

\section{Method}

\section{Participants}

Participants $(\mathrm{N}=30)$ were recruited from The New School university community, postings to the website www.craigslist.org/, and a database of participants who had taken part in other studies in 
our lab. This sample size was determined on the basis of previous studies that used similar tDCS methodology to test the effect of frontal cortex stimulation on emotion; in particular we followed Boggio and colleagues [15] who concluded on the basis of a power analysis that "20 [participants] (10 in each group) were needed to detect group difference." By recruiting 30 participants we ensured that there were at least 10 participants in each group.

To ensure that the sample included participants with the full range of handedness asymmetries, we selectively contacted left-handed and ambidextrous participants from the database. These participants were not aware that they were being contacted based on their handedness. All participants were paid in exchange for their participation. All procedures were approved by the Institutional Review Board at The New School (New York, NY, USA).

To ensure participants' safety, we excluded respondents from participation if they indicated that they were pregnant, had ever experienced an epileptic seizure, had ever sustained a stroke or other brain injury, or were taking any psychoactive drugs or medications. Additionally, we did not test anyone who reported ever having been diagnosed with depression, bipolar disorder, anxiety disorder, or schizophrenia.

One participant was excluded during the first session when low scalp impedance could not be obtained. Four additional participants did not complete all 5 sessions of the study (Rightexcitatory stimulation, $\mathrm{n}=2$; Left-excitatory stimulation, $\mathrm{n}=2$ ), and were excluded. Data were analyzed from the remaining 25 participants (Right-handers, Edinburgh Handedness Inventory $($ EHI) $>40: n=17$; Non-right-handers, EHI < 40: $n=8)$. Age and gender demographics were not collected. 


\section{Materials and Procedure}

Overview. This study took place over five consecutive days (Monday-Friday). Informed consent was obtained at the beginning of each session. Participants were paid at the end of every session, with a bonus at the fifth session.

On day 1, participants completed a battery of pre-stimulation tests, including two questionnaires measuring motivation, and two continuous measures of handedness (see below). Participants also completed a working memory task that is not relevant to the present study. After participants finished these tasks, we applied the first session of tDCS. On days 2-4, we applied tDCS after ensuring that participants had not experienced any discomfort after the previous sessions. On day 5, participants underwent tDCS and then performed the same tasks as on day 1. Participants also completed a brief adverse effects questionnaire. Upon finishing the study, participants were debriefed and encouraged to contact the experimenter if they had any further questions or experienced any discomfort.

Measuring state approach motivation. Participants completed an untimed, computerized version of the Positive and Negative Affect Scale (PANAS-X) [16]. Emotion words (e.g. "interested") appeared on the screen one at a time, and participants rated the degree to which they had experienced that emotion "during the past few days" on a scale of one ("very slightly or not at all") to five ("extremely") by pressing the numbers 1-5 on a computer keyboard. We measured approach motivation using the approach-affect subscale [16], which included the following items: enthusiastic, active, interested, strong, excited, determined, inspired, alert, proud. 
We focused on approach motivation for two reasons. First, there is disagreement among theorists as to whether "avoidance motivation" refers to the tendency to not act [18] or the tendency to act so as to withdraw or defend oneself [2]. The latter meaning of "avoidance" is relevant to our experimental hypothesis, but we are not aware of any validated, non-clinical scales that measure avoidance motivation in this sense of the tendency to perform avoidancerelated actions. Second, previous studies have found that approach motivation is more reliably associated with asymmetric cortical activation than avoidance motivation [2, 11-12; but see 19]. Therefore, here we only tested associations between EHI and the hemispheric laterality of approach motivation, as in our previous EEG study [8].

Measuring trait approach motivation. In addition to the PANAS, participants completed the Behavioral Activation System / Behavioral Inhibition System (BIS/BAS) scales [17] as a measure of dispositional trait motivation. Because the BIS/BAS scales measure stable personality traits, we did not expect that short-term tDCS would influence responses on these scales.

Handedness. Participants completed the Edinburgh Handedness Inventory as an index of manual motor asymmetries (EHI) [20]. This scale provides a continuous measurement of handedness, with scores varying from strongly left-handed (-100) to strongly right-handed (100). EHI was collected in only the first session for the first 7 participants, and in both the first and final sessions for the remaining 18 participants.

In addition, all participants completed a finger-tapping task as a measure of simple performance differences between the hands. Participants tapped with one index finger on the 
space-bar of a computer as quickly they could for $10 \mathrm{~s}$. Participants performed 3 trials with each hand for 6 trials total, alternating hand on each trial. Starting hand was randomly assigned. Asymmetry scores were calculated as $\mathrm{R}-\mathrm{L}$, where $\mathrm{R}$ and $\mathrm{L}$ are the total taps produced with the right and left index fingers in all blocks.

Transcranial Direct Current Stimulation (tDCS). Direct current stimulation was delivered using a battery-powered stimulator (Soterix Medical, New York) with two $5 \times 7 \mathrm{~cm}$ salinesoaked sponges covering the electrodes. New sponges were used for each session. In each session, direct current was applied at $2 \mathrm{~mA}$ for $20 \mathrm{~min}$. To minimize discomfort, the current slowly ramped between 0 and $2 \mathrm{~mA}$ when powering on and off. Stimulation was delivered bilaterally above dorsolateral prefrontal cortex (DLPFC) at F3-4 in the 10-20 system [21]. Because the current is likely to spread diffusely into the cortex, we do not assume that stimulation was restricted to DLPFC, but that a range of frontal areas were affected. An experimenter was in the room with the participant at all times to ensure that stimulation remained comfortable.

Stimulation was delivered using a double-blind procedure in two between-subjects conditions. Before beginning the study, a polarity-blinding box was set to either reverse the polarity of the outgoing wires, or leave polarity unchanged. This allowed both the experimenter and the participant to remain blind to the stimulation condition. Participants were randomly assigned to one of the two conditions.

In one condition, the anode was placed above F3 (left) and the cathode above F4 (right), exciting left frontal areas while inhibiting right frontal areas (Left-excitatory). ${ }^{1}$ In the second

\footnotetext{
${ }^{1}$ Although cathodal tDCS is often assumed to be inhibitory, $2 \mathrm{~mA}$ cathodal stimulation has also been found to have an effect that either is no different from sham stimulation [42] or is excitatory [43]. Our study does not rely on the
} 
condition, the anode was placed above F4 (right) and the cathode above F3 (left), exciting right while inhibiting left frontal areas (Right-excitatory). Stimulation condition remained the same across all 5 sessions for each participant. Of the participants retained in the final analysis, 10 received right-excitatory stimulation, and 15 received left-excitatory stimulation.

\section{Results}

We analyzed changes in approach emotional state as a function of tDCS polarity and the participants' EHI scores. We computed the mean of each participant's responses in the approachsubscale, separately for the pre-stimulation and post-stimulation sessions. We then computed the change in approach motivation as the difference between sessions (Session 2 - Session 1). A first set of analyses treated handedness as categorical, for comparison with previous EEG results. Of primary interest, a second set of analyses treated handedness as continuous. Following these main analyses, further analyses were conducted to confirm that tDCS had no effect on trait motivational tendencies or on handedness.

\section{Effects of tDCS on motivation with handedness coded categorically}

Lateralized tDCS changed participants' experience of approach-motivated emotions, and had opposite effects on right-handers and non-right-handers, according to a 2-by-2 ANOVA predicting the change in approach motivation as a function of tDCS polarity (right-excitatory, left-excitatory) and handedness (right-handers, non-right-handers) $(F(1,21)=35.1, p=.000007$, $\eta^{2}=1.34$, fig. 1a). In right-handers, approach emotions increased after left-excitatory stimulation

assumption that cathodal stimulation is inhibitory, only on the assumption that anodal stimulation will be more excitatory than cathodal stimulation. If anodal and cathodal stimulation were equally excitatory, then the treatment we performed would be ineffective in changing the balance of neural excitability across hemispheres, and we could not find the predicted results. 
relative to right-excitatory stimulation $(t(11.5)=2.6, p=.02, d=1.32)$. Non-right-handers showed the opposite pattern: Approach emotions increased after right-excitatory stimulation but decreased after left-excitatory stimulation $(t(4.44)=-7.8, p=.001, d=4.97)$. This categorical between-group difference is consistent with the results of an EEG study showing that greater approach motivation correlated with more left-hemisphere activity during rest in right-handers, but with more right-hemisphere activity during rest in left-handers [8].

\section{Effects of tDCS on motivation with handedness coded continuously}

For our main analysis of interest, we used linear regressions with handedness coded continuously (from -100 to $100 \mathrm{EHI}$ ) to test for covariation between manual motor asymmetries and the hemispheric lateralization of approach motivation. The effects of left- versus right-hemisphere tDCS on the experience of approach emotions covaried continuously with the individual participants' strength of handedness $(\beta=-0.14, S E=0.03, t=-5.1, p=.00004$; fig. $1 \mathrm{~b})$. After left-excitatory stimulation, stronger right-handedness was correlated with greater increase in approach emotions, and stronger left-handedness with greater decrease in approach emotions ( $\beta$ $=0.10, S E=0.02, t=4.7, p=.0004)$. By contrast, after right-excitatory stimulation stronger lefthandedness was correlated with greater increase in approach emotions, and stronger righthandedness with greater decrease $(\beta=-0.04, S E=0.02, t=-2.3, p=.05)$.

To ensure that our findings were not driven unduly by a small number of strong lefthanders, we performed additional analyses using robust regressions with Huber weights. Significance was determined with robust F-tests using the 'f.robftest' function in the $s f s m i s c$ library in R. Robust regression analyses supported the conclusions from the previous analyses. As in the standard regression analyses, robust regressions revealed a significant effect of 
handedness on approach emotions after left- excitatory stimulation $(t=4.69, F=22.042, p=$ 0.0004), a marginally significant effect of handedness on approach emotions after rightexcitatory stimulation $(t=-2.05, F=4.30, p=0.07)$, and a significant interaction of handedness and tDCS polarity $(t=-4.88, F=23.99, p=0.00008)$.
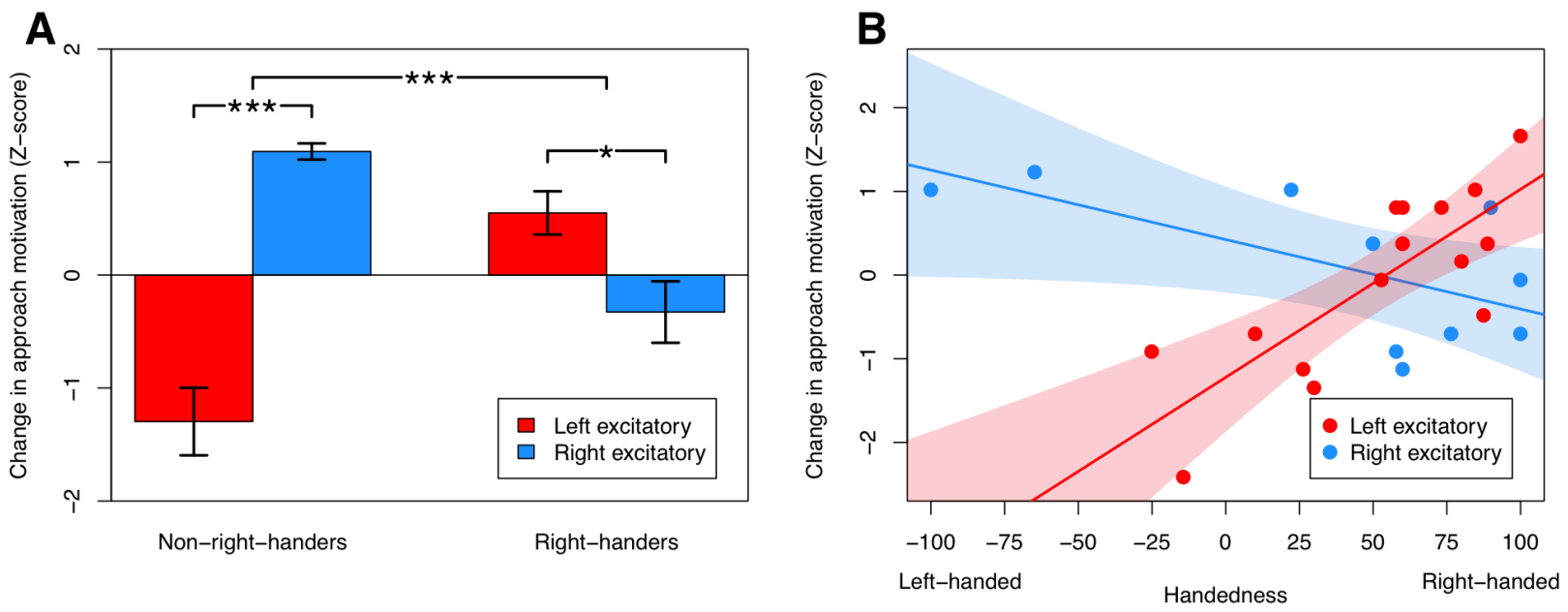

Figure 1. The laterality of approach motivation covaries with manual motor asymmetries. (A) Change in approach motivation (last day - first day) plotted separately for right- and non-righthanders, and for left- and right-excitatory tDCS. Error bars show standard error of the mean for responses averaged by subjects. $* p<.05, * * * p<.001$. (B) Change in approach motivation plotted for each participant as a function of their handedness, measured continuously (20). Each point shows the Z-transformed change in approach motivation for one participant (21). Best-fit regression lines are plotted separately for participants who received left-hemisphere excitatory stimulation and right-hemisphere excitatory stimulation, with $95 \%$ confidence intervals on the regression lines shown as shaded areas.

\section{Effects of tDCS on trait motivation}

As expected, trait approach motivation was not influenced by tDCS. No stimulation-byhandedness interaction emerged in either BAS or BIS $(p s>.9)$. This indicates that a single week of tDCS influenced only state approach motivation (measured by the PANAS scale, reported in the main analyses of the paper), but not trait approach motivation, which is believed to be a stable aspect of personality [16]. 


\section{Effects of tDCS on handedness}

As expected, tDCS had no measurable effect on participants' handedness. EHI scores were almost identical between the two testing sessions $(r=.98, n=18$ participants with EHI collected at both sessions), overall, and differences in stimulation polarity did not cause differential changes in EHI scores between sessions $(t(12.3)=-1.5, p=.16)$. Finger-tapping asymmetries were more variable $(r=.56)$, but were similarly unaffected by stimulation polarity $(t(22.8)=$ $0.28, p=.78)$. Participants' day-1 finger-tapping asymmetries did not correlate with their day-1 EHI scores $(n=25, r=-0.05, p=.8)$. EHI was used for all hypothesis testing because it was more reliable than finger tapping, and has been used more widely in previous studies.

\section{Adverse effects.}

The study was terminated prior to completion for one participant who reported a headache. Three other participants requested that the stimulation intensity be reduced for several minutes in one session; all three completed the study. Of these four participants reporting discomfort, two had received left- excitatory stimulation, and two right-excitatory stimulation. No other subjects reported notable discomfort.

\section{Discussion}

By manipulating asymmetries in cortical excitability with five sessions of tDCS, we showed that hemispheric specialization for approach motivation covaries with specialization for motor control of the dominant hand. This relationship was found both when we coded handedness categorically and when we coded it continuously. In strong right-handers, leftexcitatory tDCS led to increased approach motivation, whereas right-excitatory tDCS led to 
decreased approach motivation. In non-right-handers, by contrast, we found the opposite pattern: Right-excitatory tDCS increased approach motivation and left-excitatory tDCS decreased it. Furthermore, we found continuous covariation between hand dominance and motivation in the brain: Stronger motor asymmetries corresponded to stronger lateralization of motivation. These results support the sword and shield hypothesis [8], showing that the laterality of approach motivation covaries continuously with the laterality of manual motor control, and suggesting a functional relationship between cortical circuits for action and emotion.

These results provide a conceptual replication of our earlier EEG study showing that approach motivation was lateralized to the left hemisphere in right-handers but to the right hemisphere in left-handers [8]. Together, these studies provide a novel explanation for a large body of previous data showing a left-hemisphere bias for approach motivation [2-7,22]. This bias may have emerged in previous studies because the cortical organization of motivation reflects the organization of the motor system, and because most previous studies tested only strong righthanders for whom motor control of the "sword hand," which is used preferentially for approach actions, is strongly lateralized to the left hemisphere.

The laterality of motivation typically observed in strong right-handers has been interpreted as indicating that the left hemisphere is specialized for approach motivation. However, continuous variation in the effects of tDCS across individual subjects (fig. 1b) supports the conclusion that neither hemisphere is inherently specialized for approach motivation. Rather, approach motivation is distributed across both cerebral hemispheres, with the strength and direction of lateralization varying as a function of the consistency with which individuals tend to perform approach- and avoidance-related actions with their right and left hands. 
What other cognitive functions reverse with handedness? The complete reversal of hemispheric specialization between strong left- and right-handers sets motivation apart from other lateralized cognitive functions. Lateralization of language depends only weakly on handedness. Like most right-handers, the majority of left-handers $(\sim 75 \%)$ also have language lateralized to the left hemisphere [22-24]. According to one large-scale fMRI study $(\mathrm{N}=297)$, the correlation between handedness and language laterality is "barely above the chance level" [24]. According to another large-scale study using transcranial Doppler imaging $(\mathrm{N}=310)$, “degree of hand-preference [does] not mirror degree of language lateralization" [25].

The long-standing belief that left-handers tend to have reversed language dominance has been largely debunked. Therefore, previous proposals that have linked the cerebral laterality of approach actions or emotions to the laterality of language cannot explain the present data [7,22]. Like language, some aspects of visuospatial cognition that are clearly lateralized in right-handers are more variable in left-handers [27-28], but neither the laterality of language nor of visuospatial cognition reverses with handedness. By contrast, the laterality of dominant-hand motor control does reverse, and accordingly, so does the laterality of approach motivation.

Previous links between action and affective motivation. Some connections between affective motivation and action tendencies have been proposed previously [22], and two previous links between motivation and hand actions have been found [17,29]. Importantly, however, these previous proposals do not predict or explain the pattern of data shown here.

Cacioppo and colleagues [29] found that ideographs presented during arm flexion movements were judged to be more positive in valence than ideographs presented during arm 
extension movements. The authors predicted this result on the basis of an association between action and motivation: People tend to make flexion movements when performing approachrelated actions (e.g., eating something desirable), and extension movements when performing avoidance-related actions (e.g., rejecting something undesirable). Although these findings are important for a full understanding of connections between action and emotion, they cannot explain the cerebral laterality of motivation (nor were they intended to do so). In the original study by Cacioppo and colleagues [29] and many follow-ups, participants made flexion and extension movements with both hands simultaneously, presumably activating both hemispheres. Cacioppo and colleagues trace the neural basis of the flexion-extension effect to bilateral spinal motor neurons, not to the cerebral hemispheres [30]. The flexion-approach / extension-avoidance code appears to be a different link between action and motivation, separate from the actionmotivation link posited by the sword and shield hypothesis.

Of greater relevance, Harmon-Jones [17] showed that unilateral hand contractions increased contralateral frontal activity, and that right-hand contractions increased approachrelated emotions relative to left-hand contractions. In closing, Harmon-Jones speculated that "perhaps basic approach motivational movements are accomplished more often and/or efficiently via the right hand or right side of the body," consistent with the sword and shield hypothesis. However, Harmon-Jones's study did not test for effects of handedness and was not predicated on any differential hand use for approach vs. avoidance actions, but rather on the assumption that approach motivation is generally lateralized to the left hemisphere - an assumption challenged by the sword and shield hypothesis and by the data we present here. 
Evolution of neural systems for action and emotion. The sword and shield hypothesis may clarify not only how motivation is organized in the cerebral cortex, but also provide a potential explanation for why it is organized this way: Neural circuits for affective motivation may be built upon neural circuits that control approach- and avoidance-motivated actions. Approaching or avoiding stimuli is perhaps the most basic of all behaviors, found even in single-celled organisms that are unlikely to form affective states or intentions as humans do. We posit, therefore, that approach and avoidance actions are ontologically prior to approach and avoidance motivational states, and that neural circuits for affective motivation may reuse neural circuits that evolved primarily for performing motor actions. Affective motivational states may consist in highly abstracted motor plans, which indicate a state of readiness to perform either approach- or avoidance-related actions.

Caveats and future studies. One caveat in interpreting the results of this experiment concerns the sample size. Because neurostimulation studies carry some potential risk for subjects (and potential discomfort), sample sizes for (multi-session) tDCS studies tend to be smaller than samples for purely behavioral studies. Our sample size was consistent with established standards for this literature, however, and multiple aspects of the results suggest that this sample size was sufficient. Chiefly, a complex pattern of data was predicted a priori, and all of the predicted effects (i.e., two contrasting simple effects and a particular interaction) were statistically significant, no matter whether we analyzed the data using a categorical or a continuous coding of handedness.

To elaborate, the effect of left-excitatory stimulation, and the difference between the effects of left- and right-excitatory stimulation, remained highly significant under all three 
analysis strategies we used: ANOVA with categorical coding of handedness, regression with continuous coding of handedness, and robust regression with continuous coding of handedness. The effect of right-excitatory stimulation was significant under the first two analyses, and marginally significant under the third $(\mathrm{p}=.07)$, due to the small sample size in the right-excitatory group. Importantly, the sample size in this group was equal to the minimum sample size indicated by a previous tDCS study by Boggio and colleagues [15], which we adapted. Validating Boggio et al.'s recommendation, this sample size was sufficient to allow us to detect highly significant differences between treatment groups (i.e., left- vs. right-excitatory stimulation groups) in the predicted direction, in all analyses.

Ideally, increasing the sample size would allow us to report a highly significant effect in the right-excitatory group in all analyses, as well, and to fill in segments of the handedness continuum where the data were sparse (due to our double-blind procedure). We elected not to increase the sample size, however, for two reasons. First, all predicted effects were statistically significant in the planned sample, by ANOVA and by standard regression analyses. Second, increasing excitability in the "shield" hemisphere caused a decrease in participants' experience of positive, approach-related emotions, as predicted by the sword and shield hypothesis. For example, left-excitatory stimulation caused non-right-handers' experience of approach emotions to decrease by about a standard deviation, on average (fig. 1a, left). In short, our treatment made about half of our participants feel worse. Given this finding, it would be unethical to increase the sample size without strong justification; since all of the predicted effects were significant in the planned sample, we do not believe we had any such justification.

Consistent with current statistical practices, we do not rely on the statistical significance of these results, alone, to gauge our confidence in them, but also on their effect sizes [31]. The 
observed effects were large. In our categorical analysis, for example, the partial eta-squared value for the critical interaction was 1.34: much greater than the conventional threshold for a "large" effect, which is 0.14 . Likewise, the Cohens's $d$ value for the simple effects in righthanders and non-right-handers were 1.32 and 4.97, respectively: much larger than the conventional threshold for a "large" effect, which is 0.80 [31].

All statistical measures aside, the best indication of reliability is widely agreed to be replication $[33,34]$. Overall, the pattern of results we show here is a conceptual replication of our previous EEG study testing the sword and shield hypothesis [8]. Our effect of left excitatory stimulation on emotion in right-handers is a conceptual replication of a previous tDCS results in right-handers [15]. Finally, because our two stimulation conditions show complementary effects of handedness and hemisphericity on emotion, our right-excitatory-stimulation condition can be considered a conceptual replication of our left-excitatory-stimulation condition: two statistically independent tests of the sword and shield hypothesis. Future studies should seek to generalize these findings to a new sample of healthy participants (with proper oversight from a clinician), and also to extend them to a clinical population.

Future studies could also consider whether the relationships predicted by the sword and shield hypothesis extend to non-human animals. Many non-human animals tend to perform motivation-related actions on particular sides of their bodies [35]. For example, chicks tend to use their right eye to search the ground for food (an approach-motivated action) while their left eye scans the sky for predators (an avoidance-motivated action) [36]. Such behavioral asymmetries - whether or not they involve differential use of the hands - may correlate with the lateralization of approach motivation in non-human animals' brains. 


\section{Potential clinical implications of the body-specific organization of motivation. Our results} support the body-specificity hypothesis: Individuals with different kinds of bodies, who interact with the environment in systematically different ways, develop corresponding differences in their brains and minds [37]. In addition to addressing basic scientific questions, our findings raise questions about clinical treatments for psychiatric disorders.

Individual differences in the neural organization of motivation may have urgent implications for the safety and success of neural therapies for anxiety disorders and depression. Clinicians use tDCS or transcranial magnetic stimulation (TMS) to stimulate left frontal areas in order to promote positive, approach-related emotions [15,38-40]. Yet this treatment is predicated on the assumption that the left hemisphere is specialized for approach motivation. Our results indicate that this assumption may be false for non-right-handed people, who constitute about 40$50 \%$ of the general population (depending on how handedness categories are defined) [41]. Given that the present study tested only healthy non-clinical volunteers, conclusions about clinical treatments would be premature. Nevertheless, these results suggest that neurostimulation treatments that benefit strong right-handers could be ineffective or detrimental for everyone else, and that neural therapies for common psychiatric disorders should be tailored to the specifics of people's bodies. 


\section{References}

1. Casasanto D, Bottini, R. 2014 Spatial language and abstract concepts. WIREs Cognitive Science 5, 139-149. (doi:10.1002/wcs.1271)

2. Amodio DM, Master SL, Yee CM, Taylor SE. 2008 Neurocognitive components of the behavioral inhibition and activation systems: Implications for theories of self-regulation. Psychophysiology 45, 11-19. (doi:10.1111/j.1469-8986.2007.00609.x)

3. Berkman ET, Lieberman MD. 2010 Approaching the bad and avoiding the good: Lateral prefrontal cortical asymmetry distinguishes between action and valence. Journal of Cognitive Neuroscience 22, 1970-1979. (doi:10.1162/jocn.2009.21317)

4. Coan JA, Allen JJ. 2004 Frontal EEG asymmetry as a moderator and mediator of emotion. Biological Psychology 67, 7-50. (doi:10.1016/j.biopsycho.2004.03.002)

5. Davidson RJ, Ekman P, Saron CD, Senulis JA, Friesen WV. 1990 Approach-withdrawal and cerebral asymmetry: Emotional expression and brain physiology: I. Journal of Personality and Social Psychology 58, 330-341. (doi:10.1037//0022-3514.58.2.330)

6. Harmon-Jones E, Gable PA, Peterson CK. 2010 The role of asymmetric frontal cortical activity in emotion-related phenomena: A review and update. Biological Psychology 84, 451-462. (doi:10.1016/j.biopsycho.2009.08.010)

7. Reuter-Lorenz PA, Givis RP, Moscovitch M. 1983 Hemispheric specialization and the perception of emotion: Evidence from right-handers and from inverted and non-inverted left-handers. Neuropsychologia 21, 687-692. (doi:10.1016/0028-3932(83)90068-4)

8. Brookshire G, Casasanto D. 2012 Motivation and motor control: hemispheric specialization for approach motivation reverses with handedness. PLoS One 7, e36036. (doi:10.1162/jocn.2009.21317) 
9. Casasanto D. 2009 Embodiment of abstract concepts: good and bad in right-and lefthanders. Journal of Experimental Psychology: General 138, 351-367. (doi:10.1037/a0015854)

10. Coren S. 1992 Handedness, traffic crashes, and defensive reflexes. American Journal of Public Health 82, 1176-1177. (doi:10.2105/ajph.82.8.1176-b)

11. Coan JA, Allen JJ. 2003 Frontal EEG asymmetry and the behavioral activation and inhibition systems. Psychophysiology 40, 106-114. (doi:10.1111/1469-8986.00011)

12. Harmon-Jones E, Allen JJ. 1997 Behavioral activation sensitivity and resting frontal EEG asymmetry: Covariation of putative indicators related to risk for mood disorders. Journal of Abnormal Psychology 106, 159. (doi:10.1037//0021-843x.106.1.159)

13. Sutton SK, Davidson RJ. 1997 Prefrontal brain asymmetry: A biological substrate of the behavioral approach and inhibition systems. Psychological Science 8, 204-210. (doi:10.1111/j.1467-9280.1997.tb00413.x)

14. Nitsche MA, Cohen LG, Wassermann EM, Priori A, Lang N, Antal A, Paulus W, Hummel F, Boggio PS, Fregni F, Pascual-Leone A. 2008 Transcranial direct current stimulation: state of the art 2008. Brain Stimulation 1, 206-223. (doi:10.1016/j.brs.2008.06.004)

15. Boggio PS, Rigonatti SP, Ribeiro RB, Myczkowski ML, Nitsche MA, Pascual-Leone A, \& Fregni F. 2008 A randomized, double-blind clinical trial on the efficacy of cortical direct current stimulation for the treatment of major depression. International Journal of Neuropsychopharmacology 11, 249-254. (doi:10.1017/S1461145707007833)

16. Watson D, Clark LA. 1999 The PANAS-X: Manual for the positive and negative affect schedule-expanded form. (University of Iowa) 
17. Harmon-Jones E. 2006 Unilateral right-hand contractions cause contralateral alpha power suppression and approach motivational affective experience. Psychophysiology 43, 598603. (doi:10.1111/j.1469-8986.2006.00465.x)

18. Carver CS, White TL. 1994 Behavioral inhibition, behavioral activation, and affective responses to impending reward and punishment: The BIS/BAS Scales. Journal of personality and social psychology 67, 319-333. (doi:10.1037/0022-3514.67.2.319)

19. Shackman AJ, McMenamin BW, Maxwell JS, Greischar LL, Davidson RJ. 2009 Right dorsolateral prefrontal cortical activity and behavioral inhibition. Psychological science 20, 1500-6. (doi:10.1111/j.1467-9280.2009.02476.x)

20. Oldfield RC. 1971 The assessment and analysis of handedness: the Edinburgh inventory. Neuropsychologia 9, 97-113. (doi:10.1016/0028-3932(71)90067-4)

21. DaSilva AF, Volz MS, Bikson M, Fregni F. 2011 Electrode positioning and montage in transcranial direct current stimulation. JoVE 51, e2744. (doi:10.3791/2744)

22. Kinsbourne M. 1978 Biological determinants of functional bisymmetry and asymmetry. In M. Kinsbourne (Ed.), Asymmetrical Function of the Brain (3-16), Cambridge University Press.

23. Pujol J, Deus J, Losilla JM, Capdevila A. 1999 Cerebral lateralization of language in normal left-handed people studied by functional MRI. Neurology 52, 1038-1038. (doi:10.1212/wnl.52.5.1038)

24. Knecht S, Dräger B, Deppe M, Bobe L, Lohmann H, Flöel A, Ringelstein EB, Henningsen H. 2000 Handedness and hemispheric language dominance in healthy humans. Brain 123, 2512-2518. (doi:10.1093/brain/123.12.2512) 
25. Mazoyer B, Zago L, Jobard G, Crivello F, Joliot M, Perchey G, Mellet E, Petit L, Tzourio-Mazoyer N. 2014 Gaussian mixture modeling of hemispheric lateralization for language in a large sample of healthy individuals balanced for handedness. PLoS One 9, e101165. (doi:10.1371/journal.pone.0101165)

26. Somers M, Aukes MF, Ophoff RA, Boks MP, Fleer W, Kahn RS, Sommer IE. 2015 On the relationship between degree of hand-preference and degree of language lateralization. Brain and Language 144, 10-15. (doi:10.1016/j.band1.2015.03.006)

27. Jager G, Postma A. 2003 On the hemispheric specialization for categorical and coordinate spatial relations: A review of the current evidence. Neuropsychologia 41, 504515. (doi:10.1016/s0028-3932(02)00086-6)

28. Willems RM, Peelen MV, Hagoort P. 2010 Cerebral lateralization of face-selective and body-selective visual areas depends on handedness. Cerebral Cortex 20, 1719-1725. (doi:10.1093/cercor/bhp234)

29. Cacioppo JT, Priester JR, Berntson GG. 1993 Rudimentary determinants of attitudes: II. Arm flexion and extension have differential effects on attitudes. Journal of personality and social psychology 65, 5-17. (doi:10.1037/0022-3514.65.1.5)

30. Norman GJ, Norris CJ, Gollan J, Ito TA, Hawkley LC, Larsen JT, Cacioppo JT, Berntson GG. 2011 Current emotion research in psychophysiology: The neurobiology of evaluative bivalence. Emotion Review 3, 349-59. (doi:10.1177/1754073911402403)

31. Cumming G. 2014 The new statistics: Why and how. Psychological science 25, 7-29. (doi:10.1177/0956797613504966)

32. Cohen J. 1988 Statistical power analysis for the behavioral sciences. Hilsdale. NJ: Lawrence Earlbaum Associates. 
33. Cohen, J. (1994). The earth is round ( $<$.05): Rejoinder. American Psychologist 50, 997-1103. (doi:10.1037/0003-066X.50.12.1103)

34. Popper K. 1959 The logic of scientific discovery. Routledge.

35. Rogers LJ, Vallortigara G, Andrew RJ. 2013 Divided Brains: The Biology and Behaviour of Brain Asymmetries. Cambridge University Press, New York.

36. Vallortigara G. 2000 Comparative neuropsychology of the dual brain: A stroll through animals' left and right perceptual worlds. Brain and Language 73, 189-219. (doi:10.1006/brln.2000.2303)

37. Casasanto D. 2011 Different bodies, different minds: The body-specificity of language and thought. Current Directions in Psychological Science 20, 378-383. (doi:10.1177/0963721411422058)

38. Gross M, Nakamura L, Pascual-Leone A, Fregni F. 2007 Has repetitive transcranial magnetic stimulation (rTMS) treatment for depression improved? A systematic review and meta-analysis comparing the recent vs. the earlier rTMS studies. Acta Psychiatrica Scandinavica 116, 165-173. (doi:10.1111/j.1600-0447.2007.01049.x)

39. Murphy DN, Boggio P, Fregni F. 2009 Transcranial direct current stimulation as a therapeutic tool for the treatment of major depression: insights from past and recent clinical studies. Current Opinion in Psychiatry 22, 306-311. (doi:10.1097/yco.0b013e32832a133f)

40. Nitsche MA, Boggio PS, Fregni F, Pascual-Leone A. 2009 Treatment of depression with transcranial direct current stimulation (tDCS): a review. Experimental Neurology 219, 14-19. (doi:10.1016/j.expneurol.2009.03.038) 
41. Annett M. 2004 Hand preference observed in large healthy samples: Classification, norms and interpretations of increased non-right-handedness by the right shift theory. British Journal of Psychology 95, 339-53. (doi:10.1348/0007126041528130)

42. Wiethoff S, Hamada M, Rothwell JC. 2014 Variability in response to transcranial direct current stimulation of the motor cortex. Brain stimulation 7, 468-75. (doi:10.1016/j.brs.2014.02.003)

43. Batsikadze G, Moliadze V, Paulus W, Kuo MF, Nitsche MA. 2013 Partially non-linear stimulation intensity-dependent effects of direct current stimulation on motor cortex excitability in humans. The Journal of Physiology. 591,1987-2000. (doi:

10.1113/jphysiol.2012.249730) 